\title{
Optimal wavelet denoising for smart bio-monitor systems
}

\author{
Sheila R. Messer ${ }^{a}$, John Agzarian ${ }^{b}$ and Derek Abbott ${ }^{a}$ \\ ${ }^{a}$ Centre for Biomedical Engineering (CBME) \\ Department of Electrical and Electronic Engineering \\ Adelaide University, SA 5005, Australia \\ ${ }^{b}$ Hampstead Medical Clinic, Hampstead Gardens, SA 5086, Australia
}

\begin{abstract}
Future smart-systems promise many benefits for biomedical diagnostics. The ideal is for simple portable systems that display and interpret information from smart integrated probes or MEMS-based devices. In this paper, we will discuss a step towards this vision with a heart bio-monitor case study. An electronic stethoscope is used to record heart sounds and the problem of extracting noise from the signal is addressed via the use of wavelets and averaging. In our example of heartbeat analysis, phonocardiograms (PCGs) have many advantages in that they may be replayed and analysed for spectral and frequency information. Many sources of noise may pollute a PCG including foetal breath sounds if the subject is pregnant, lung and breath sounds, environmental noise and noise from contact between the recording device and the skin. Wavelets can be employed to denoise the PCG. The signal is decomposed by a discrete wavelet transform. Due to the efficient decomposition of heart signals, their wavelet coefficients tend to be much larger than those due to noise. Thus, coefficients below a certain level are regarded as noise and are thresholded out. The signal can then be reconstructed without significant loss of information in the signal. The questions that this study attempts to answer are which wavelet families, levels of decomposition, and thresholding techniques best remove the noise in a PCG. The use of averaging in combination with wavelet denoising is also addressed. Possible applications of the Hilbert Transform to heart sound analysis are discussed.
\end{abstract}

Keywords: Smart bio-monitors, wavelets, denoising, phonocardiogram, heart sound analysis, heartbeat analysis, Hilbert transform

\section{INTRODUCTION}

The stethoscope and human ear have their limitations in diagnosing heart defects and conditions. Modern technology has developed new tools which are capable of revealing information that traditional tools such as the stethoscope alone cannot. For example, digital stethoscopes have been developed which have the capacity to record and to replay the heartbeat sound recordings otherwise known as phonocardiograms(PCGs). The PCG is a particularly useful diagnostic tool because the graphic recordings show timings and relative intensities of heartbeat sounds and may reveal information that the human ear cannot. ${ }^{1-3}$ With the aid of computers, the PCG data may be stored, managed, and manipulated for frequency and spectral content.

Electrocardiograms (ECGs), which reveal the electrical activity of the heart, and echocardiography, which uses ultrasound waves to create an image of the heart, are other methods that are used to access the condition the heart. All of the mentioned techniques are non-invasive, but each presents a separate set of issues and challenges.

With the advent of echocardiography, auscultation and phonocardiography have become less important. However, with the aid of computers and digital signal processing techniques, PCGs may reveal important information. ${ }^{1}$

The ECG is not normally used unless a problem has previously detected by auscultation (listening to the heart sounds) because the time required to set up an ECG recording is great enough that it is not used as a standard test. However, PCGs are easily obtained by placing the stethoscope against the skin. The current problem with many PCG systems is noise from breath sounds, contact of the stethoscope with the skin, foetal heart sounds if the subject is pregnant, and ambient noise may corrupt the heartbeat signals.

Further author information:

S.R.M.: E-mail: smesser@eleceng.adelaide.edu.au

D.A.: E-mail: dabbott@eleceng.adelaide.edu.au 
The PCG would be a much more useful diagnostic tool if unwanted noise was removed clearly revealing the heartbeat sound. The current study examines methods of removing the noise from the PCG namely using wavelet analysis and averaging.

Although it is not exactly known what produces each of the four heart sounds heard through a stethoscope, they are likely produced by a number of sources including the opening and closing of valves, vibrations of the cardiac structure, and acceleration and deceleration of blood. ${ }^{1,4}$ The first and second heart sounds ("lub-dub") are the two that are generally heard by the human ear, and are usually the most visible on PCG. They may be seen in Figure 1 (a) with the first heart sound, S1, occurring at about 0.1 seconds and the second heart sound, S2, happening at about 0.4 seconds.

An electronic stethoscope is used to record heart sounds. Various digital signal processing tools namely wavelets are employed remove noise from signal. The remainder of this article will introduce the reader to basic wavelet theory in relation to heart sounds and the methods of denoising heart sounds which were investigated.
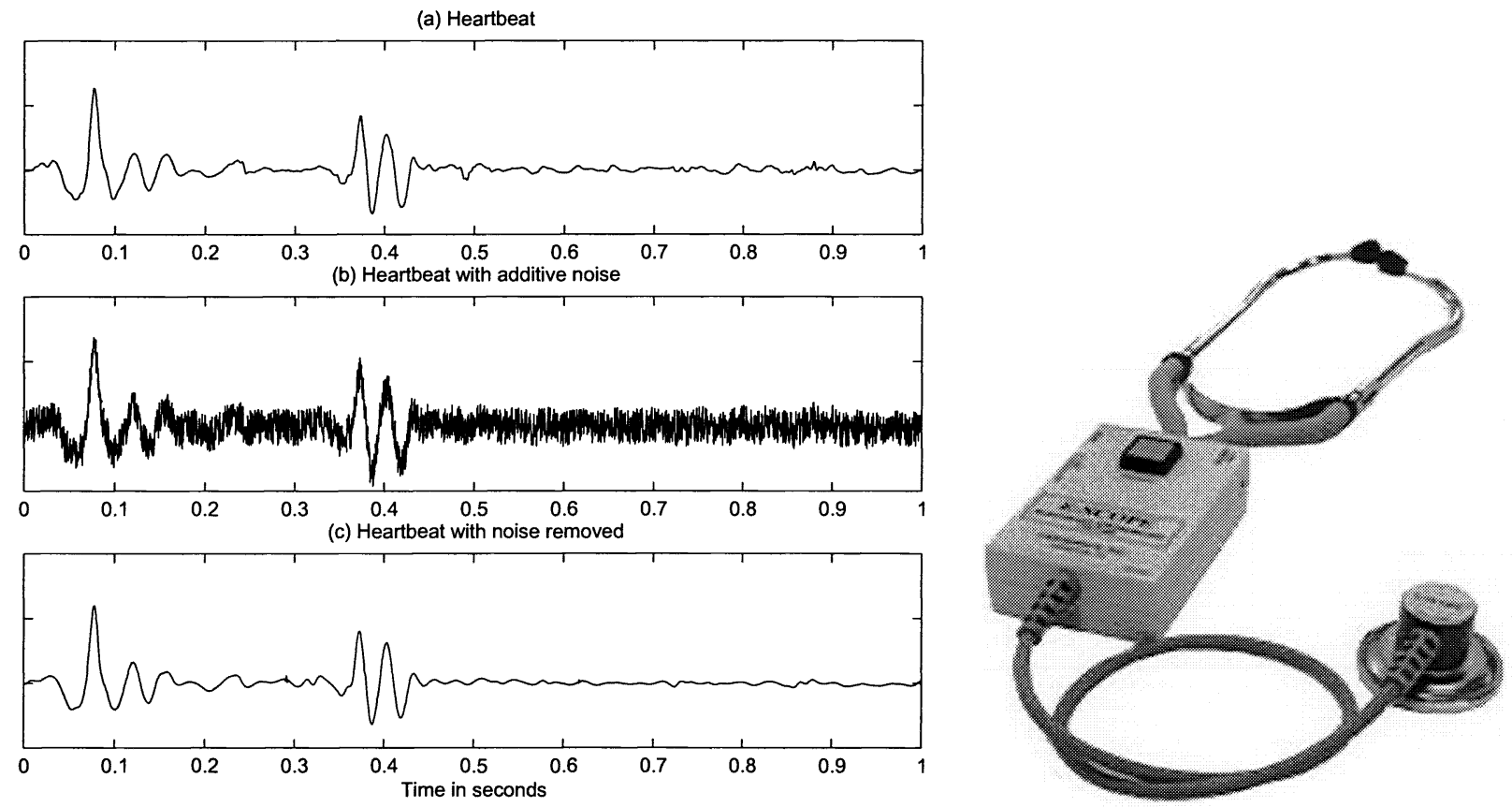

Figure 1. This figure shows the principle of denoising a heartbeat. (a) Is the characteristic heartbeat signal (b) the heartbeat with $1 \mathrm{~dB}$ of additive white noise (c) the heartbeat with noise removed using wavelet analysis

Figure 2. The Escope from Cardionics is an electronic stethoscope that is used to record heart sounds.

Wavelets may be used to denoise the PCG as shown in Figure 1. The signal is decomposed by a discrete wavelet transform. Because of the efficient decomposition of heart signals, their wavelet coefficients tend to be much larger than those due to noise. Thus, coefficients below a certain level are regarded as noise and thresholded out. The signal is then reconstructed without significant loss of information. The questions that this study attempts to answer are which wavelet families, levels of decomposition, and thresholding techniques best remove the noise in a PCG. This study is an extension of the work described by Maple $e t$ al. ${ }^{5}$

Averaging may also be used to produce a characteristic heartbeat. ${ }^{4}$ Although heartbeats are considered nonstationary signals, they are periodic in the sense that heartbeats regularly repeat. Over short periods of time, heartbeats have the same statistical properties. Thus, the signal may be considered quasi-stationary over a short period of time. This study examines the use of averaging in combination with wavelet analysis to remove noise from a PCG. 


\section{EQUIPMENT}

An electronic stethoscope outputs the heart sound as an analogue signal. This analogue signal is converted to a digital signal and stored on the computer for further use. The electrical activity of the heart is also simultaneously recorded to serve as a reference signal.

\subsection{The Escope}

The electronic stethoscope used is the Escope from Cardionics which may be seen in Figure 2. The Escope outputs an analogue signal which is converted to a digital signal by the WIN-30D A/D converter. Escope specifications may be seen in Table 1 .

\begin{tabular}{|l|l|}
\hline Audio gain & $27 \mathrm{~dB} @ 200 \mathrm{~Hz}$ \\
\hline Frequency response & $\begin{array}{l}\text { Heart sounds: } 100 \text { to } 240 \mathrm{~Hz}(-3 \mathrm{~dB}) \text { and } 45 \text { to } 900 \mathrm{~Hz}(-20 \mathrm{~dB}) \\
\text { Breath sounds: } 125 \text { to } 350 \mathrm{~Hz}(-3 \mathrm{~dB}) \text { and } 50 \text { to } 2000 \mathrm{~Hz}(-20 \mathrm{~dB})\end{array}$ \\
\hline Maximum output & $124 \mathrm{~dB}$ SPL, undistorted \\
\hline Microphone & Sound pressure type with electret microphone element located in chest piece \\
\hline Weight & 255 grams \\
\hline Length & $87.6 \mathrm{~cm}$ from chest piece to binaural earpieces \\
\hline
\end{tabular}

Table 1. Specifications for the Escope

\subsection{The ECG Recording System}

The ECG is also recorded simultaneously to give additional information and to serve as a reference signal. The purpose of the ECG system is to detect the electrical pulse generated which causes the heart to contract. To obtain the ECG, the voltage difference between two electrodes, in contact with the skin, is measured. The electrodes use a conductive gel and a pad with a fairly large surface area to give a highly conductive contact between the skin and the ECG leads. The electrodes may be positioned any number of ways. A simple configuration is used with the +ve slightly below the right collarbone, the -ve on the sixth rib under the left arm, and the ground lead on the right wrist.

The body has a high impedance which could cause problems. The problem of high impedance of the body was resolved by connecting the electrodes directly to the +ve inputs of the operational amplifier. Next, the two signals are passed through a difference amplifier. A high pass filter is used to remove any DC component present. At the last stage of the signal processing, there is an anti-aliasing filter present at $2 \mathrm{kHz}$. The output impedance is $10 \mathrm{k} \Omega$. The output is passed to the WIN30D A/D card connected to the PC.

\subsection{The WIN-30D}

An analogue to digital converter card, the WIN-30D, is used to convert the analogue signal to a digital signal which may be stored on a computer. The analogue signals are sampled at 2500 samples/sec with 12 -bit resolution. See Table 2 for more information about the WIN-30D A/D converter.

\section{WAVELET THEORY}

Wavelet theory dates back to the work of Joseph Fourier, but most of the advances in the field have been made since the 1980's. This section gives a review of basic theory needed to understand wavelet denoising.

\subsection{Fourier Analysis}

In 1822, Joseph Fourier discovered that any periodic function could be represented as an infinite sum of periodic complex exponential functions. ${ }^{6}$ The inclusive property of only periodic functions was later extended to any discrete time function. The Fourier Transform (FT) converts a signal expressed in the time domain to a signal expressed in the frequency domain. The FT representation of a signal may be seen in Figure 3(a). The FT is widely used and usually implemented in the form of the Fast FT algorithm. The mathematical definition of the FT is given below

$$
X(f)=\int_{\infty}^{-\infty} x(t) e^{-j 2 \pi f t} d t
$$




\begin{tabular}{|l|l|}
\hline A/D resolution & 12 Bits \\
\hline Nonlinearity & $\begin{array}{l}\text { Less than }+/-1 \\
\text { LSB }\end{array}$ \\
\hline $\begin{array}{l}\text { A/D full scale input } \\
\text { ranges }\end{array}$ & $\begin{array}{l}\text { Unipolar range: } \\
0 \text { to }+5 \mathrm{~V} \\
\text { Bipolar range: } \\
-5 \text { to }+5 \mathrm{~V}\end{array}$ \\
\hline $\begin{array}{l}\text { Number of A/D } \\
\text { Inputs }\end{array}$ & $\begin{array}{l}16 \text { single ended } \\
\text { A/D throughput } \\
\text { rate }\end{array}$ \\
\hline
\end{tabular}

Table 2. WIN-30D Characteristics

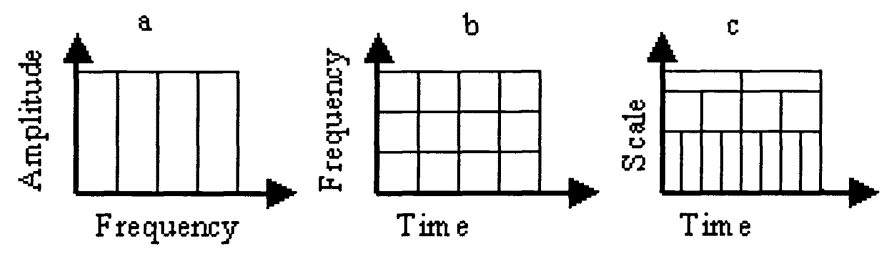

Figure 3. Comparison of a signal represented in different domains with (a) corresponding to the Fourier transform representation, (b) representing the short time Fourier transform, and (c) the wavelet transform

The time domain signal $x(t)$ is multiplied by a complex exponential at a frequency $f$ and integrated over all time. In other words, any discrete time signal may be represented by a sum of sines and cosines which are shifted and are multiplied by a coefficient that changes their amplitude. $X(f)$ are the Fourier coefficients which are large when a signal contains a frequency component around the frequency $f$. The peaks in a plot of the FT of a signal correspond to dominant frequency components of the signal.

The Fast Fourier Transform (FFT) is widely used, perhaps even too widely used. Yves Meyer states, ${ }^{7}$ "Because the FFT is very effective, people have used it in problems where it is not useful-the way Americans use cars to go half a block..."

Fourier analysis is simply not effective when used on non-stationary signals because it does not provide frequency content information localised in time. Most real world signals exhibit non-stationary characteristics (such as heart sound signals), thus Fourier analysis is not adequate.

\subsection{Short Time Fourier Transform (STFT)}

The problem with Fourier analysis is the fact that it does not matter when frequency components appear in a signal because the signal is integrated over all time in Equation 1. Thus, the frequency content of the signal is known but its location in time is not known.

In an effort to answer this problem, the STFT was developed in 1946 by Denis Gabor. ${ }^{8}$ The STFT analyses a small section of the signal at a time which is known as windowing. The STFT is a compromise between the time and frequency representation of a signal providing information on the frequency content and when it occurs. The tradeoff is between rather imprecise time and frequency resolution, which is determined by the window size. The STFT representation of a signal may be seen in $3(\mathrm{~b})$. The mathematical representation of the STFT is

$$
\operatorname{STFT}_{\mathrm{x}}^{(\mathrm{w})}\left(\mathrm{t}^{\prime}, \mathrm{x}\right)=\int_{\mathrm{t}}\left[\mathrm{x}(\mathrm{t}) \mathrm{w}^{*}\left(\mathrm{t}-\mathrm{t}^{\prime}\right)\right] \mathrm{e}^{-\mathrm{j} 2 \pi \mathrm{ft}} \mathrm{dt}
$$

where $x(t)$ is the signal and $w(t)$ is the windowing function which is translated by a certain amount denoted as $t^{\prime}$. The windowing process translates the complex conjugate of the window function along the length of the signal while multiplying the signal and windowing function at different points in time. The function of the exponential component in Equation 2 is to convert the product of multiplication of the signal and windowing function from the time domain to the frequency domain.

The problem with the STFT is a compromise in resolution. The smaller the window used, the better quickly changing components are picked up, but slowly changing details are not detected very well. If a larger window is used, lower frequencies may be detected, but localisation in time becomes worse. 


\subsection{The Wavelet Transform (WT)}

The Wavelet Transform was developed as a method to obtain simultaneous, high resolution time and frequency information about a signal. The term "wavelet" was first mentioned in 1909 in a thesis by Alfred Haar, ${ }^{8}$ although the progress in the field of wavelets has been relatively slow until the 1980's when scientists and engineers from different fields realised they were working on the same concept and began collaborating. ${ }^{7}$

The WT presents an improvement over the STFT because it obtains good time and frequency resolution simultaneously by using a variable sized window region (the wavelet) instead of a constant window size. Because the wavelet may be dilated or compressed as is seen in Figure 3(c), different features of the signal are extracted. While a narrow wavelet extracts high frequency components, a stretched wavelet picks up on the lower frequency components of the signal.

A wavelet is a signal of limited duration that has an average value of zero. Examples of wavelets used in this study may be seen in Figure 4.
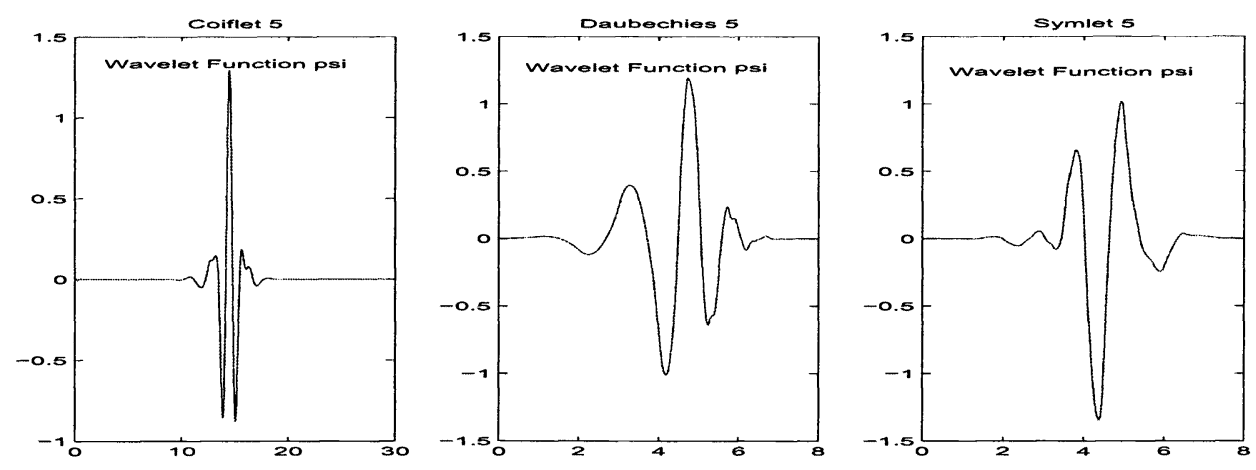

Figure 4. Examples of wavelets used in this study

The mathematical description of the Continuous Wavelet Transform (CWT) is described by

$$
C W T_{x}^{\Psi}(\tau, s)=\Psi_{x}^{\psi}(\tau, x)=\frac{1}{\sqrt{|s|}} \int x(t) \psi^{*}\left(\frac{t-\tau}{s}\right) d t
$$

The scale, $s$, of the wavelet may conceptually be considered the inverse of the frequency. As seen in Figure 3 (c), the wavelet is compressed if the scale is low and dilated if the scale is high. Because the WT is computed in terms of scale instead of frequency, plots of the WT of a signal are displayed as time versus scale.

The process of computing the CWT is very similar to that of the STFT. The wavelet is compared to a section at the beginning of a signal. A number is calculated showing how closely correlated the wavelet and signal section are. The wavelet is shifted right and and the process is repeated until the whole signal is covered. The wavelet is scaled and the previous process is repeated for all scales.

The CWT reveals much detail about a signal, but because all scales are used to compute the WT, the computation time required can be enormous. Therefore, the Discrete Wavelet Transform (DWT) is normally used. The DWT calculates the wavelet coefficients at discrete intervals of time and scale instead of at all scales. The DWT requires much less computation time than the CWT without much loss in detail. With the DWT, a fast algorithm is possible which possesses the same accuracy as other methods. The algorithm makes use of the fact that if scales and positions are chosen based on powers of two (dyadic scales and positions) the analysis is very efficient. Because the algorithm possesses the same accuracy as other methods, this method is often used and is used in the current study. 


\section{DENOISING OF HEARTBEATS}

\subsection{Signal Decomposition and Reconstruction}

The MATLAB Wavelet Toolbox provides built in routines for using the DWT to decompose a signal into wavelet coefficients and then to reconstruct the signal using the Inverse Discrete Wavelet Transform (IDWT). Many wavelet families are available in the toolbox. However, in the current study only orthogonal wavelets are examined because they allow for perfect reconstruction of a signal. The process used by MATLAB in decomposing and reconstructing a signal applies a series of highpass and lowpass filters in succession. This procedure was developed by Mallat in 1988, and is a well known signal processing tool called the two-channel subband coder. ${ }^{9}$ The signal is passed through highpass and lowpass filters and downsampled to keep the original number of datapoints. This procedure results in details which are low scale, high frequency elements of the signal, and approximations which are high scale, low frequency elements of the signal. This decomposition can be performed for many levels with the decomposition process being iterated for successive approximations. A more detailed description of the process is contained in the Wavelet Toolbox Users Guide ${ }^{8}$ and in Introduction to Wavelets and Wavelet Transforms: A Primer. ${ }^{10}$

\subsection{White Noise Removal by Thresholding}

A classic problem with most noise removal methods is actually knowing which part of the signal the noise is and how to classify the signal. With wavelet denoising, it is not necessary to know which part of the signal is white noise. David Donoho and Iain Johnstone proved mathematically that if a special kind of orthogonal basis existed, then it would do the best job at removing white noise from a signal. Then, in 1990, Donoho conversed with Domonique Picard and Gerard Kerkyachaian and realized that they had found the answer to the question of white noise removal using wavelets. ${ }^{7}$ The wavelet transform is applied to the signal and all coefficients below a certain size are discarded. ${ }^{7}$ This technique makes use of the fact that some of the decomposed wavelet coefficients correspond to signal averages and others are associated with details on the original signal. If the smaller details are eliminated from the signal decomposition, the original signal can be extracted from the remaining coefficients and the main signal characteristics will remain intact because an orthogonal wavelet transform compresses the "energy" of the signal into a few large components and the white noise is very disordered so it is scattered throughout the transform in small coefficients. ${ }^{7,11}$

\subsection{Optimal Parameter Selection For Wavelet Denoising of PCGs}

When using wavelets to denoise PCGs, there are many factors that must be considered. Examples of these choices are which wavelet, level of decomposition, and thresholding methods to use.

\subsubsection{Choosing the Wavelets To Use}

In general, the more a wavelet resembles the signal, the better it denoises the signal. However, because it is very difficult and time-consuming to actually create a useful wavelet, the wavelets which will be considered for use will be previously defined.

MATLAB provides several families of wavelets including the Morlet, Mexican hat, Meyer, Haar, Daubechies, Symlets, Coiflets and Spline biorthogonal wavelets and provides further documentation about these wavelet families. ${ }^{8}$ In order to obtain perfect reconstruction results, only orthogonal wavelets will be considered. The orthogonal wavelet transform ${ }^{*}$ has certain benefits. It is very concise, allows for perfect reconstruction of the original signal and is not very difficult to calculate. ${ }^{8}$ The ease of calculation exists because each wavelet coefficient is calculated with only one scalar product of the signal and the wavelet. ${ }^{7}$ Being computationally inexpensive, orthogonal wavelets allow a transform to be computed which contains the same number of points as the original signal. ${ }^{7}$ Wavelets that have the properties of orthogonality and a scaling function $\phi$ allow the use of the fast algorithm. The wavelets which satisfy these criteria are the Haar, Daubechies, Coiflets, and Symlets.

In MATLAB, the Daubechies family of wavelets consists of 45 wavelets. The Haar wavelet is the first and most simple wavelet in this family. The Daubechies family of wavelets is not explicitly mathematically defined except for the Haar wavelet. Most Daubechies wavelets are not symmetrical.

The Symlet family of 45 wavelets is more symmetrical than the Daubechies but is still not exactly symmetric. Ingrid Daubechies modified her family of wavelets so that their symmetry would be increased while they remained simple. Only the first 15 wavelets in this family were examined because their increasing complexity requires much

\footnotetext{
*A wavelet basis is orthogonal if every wavelet is at ninety degrees to every other wavelet.
} 
more computation time. For example, on the same computer under similar conditions, the denoising of the same sample with a Daubechies 15 wavelet took about .27 seconds and a Symlet 15 took about 1.27 seconds and using a Daubechies 25 wavelet it took about .50 seconds of computation time but increased to 54.38 seconds using a Symlet 25 wavelet.

The Coiflet family of 5 wavelets was built by Daubechies at the request of Coifman. These wavelets are more symmetrical than the Daubechies wavelets.

For each decomposition level, the signal is passed through quadrature filter banks resulting in approximations and details. This decomposition can be performed for many levels with the decomposition process being iterated for successive approximations. For every wavelet, decomposition levels of $N$ from 1 to 10 were tested.

\subsubsection{Choosing Threshold Parameters}

The two common methods of thresholding a signal are soft thresholding and hard thresholding which are used in the MATLAB Wavelet Toolbox. ${ }^{8}$ The definitions of the two methods of thresholding are given below where $x_{0}$ represents the threshold and $x$ denotes signal. ${ }^{8}$

$$
\begin{aligned}
\text { Hard Thresholding: thresholded signal } & =x \text { for }|x|>x_{0} \text {, and } 0 \text { for }|x| \leq x_{0} . \\
\text { Soft Thresholding: thresholded signal } & =\operatorname{sign}(x)\left(|x|-x_{0}\right) \text { for }|x|>x_{0} \text {, and } 0 \text { for }|x| \leq x_{0} .
\end{aligned}
$$

In other words, hard thresholding is setting the elements whose absolute values are less than the threshold to zero. For soft thresholding, the elements whose absolute values are lower than the threshold are set to zero, and then the nonzero coefficients remaining are shrunk towards zero.

Although hard thresholding is the simplest method, soft thresholding can produce better results than hard thresholding. The reason for this is that hard thresholding may cause discontinuities at $\pm x_{0}$ because those values less than the threshold are set to zero. ${ }^{8}$

\begin{tabular}{|l|l|}
\hline Rule Name & Description \\
\hline rigrsure & selection using the principle of Stein's Unbiased Risk Estimate (SURE) \\
\hline sqtwolog & fixed form threshold equal to the squareroot of two times the logarithm of the length of the signal \\
\hline heursure & selection using a mixture of the first two options mentioned \\
\hline minimaxi & threshold selection using the minimax principle \\
\hline
\end{tabular}

Table 3. Threshold selection rules

There are four threshold selection rules that are available to use with the Wavelet Toolbox ${ }^{8}$ and are listed in Table 3. These threshold selection rules use statistical regression of the noisy coefficients over time to obtain a non-parametric estimation of the reconstructed signal without noise. For the soft threshold estimator in the first method, a threshold selection rule which is based on Stein's Unbiased Estimate of Risk, is used. An estimation of risk for a certain threshold value $x_{0}$ is obtained, and then by minimizing the risks in $x_{0}$, a selection of the threshold value is obtained. The second method uses a fixed form threshold which results in minimax performance multiplied by a factor proportional to logarithm of the length of the signal. The third method is a combination of the first and second methods. If the signal-to-noise ratio is very small (for the third method), the SURE estimate is very noisy. If the signal-to-noise ratio is very small and the SURE estimate is very noisy, then the fixed form threshold is used. The fourth method uses a fixed threshold which is chosen to give minimax performance for mean square error. The minimax principle is used in the field of statistics to achieve the "minimum of the maximum mean square error." Figure 5 shows the results of each of the four threshold selection rules applied to a noisy signal.

The basic noise model used for wavelet denoising in the MATLAB Wavelet Toolbox is: $s(n)=f(n)+\sigma e(n)$ where $s$ is the complete signal, $f$ is the signal without noise, $e$ is the noise, $\sigma$ is the strength of the noise, and time $n$ is equally spaced. ${ }^{8}$ The objective of the denoising process is to suppress the noisy part of the signal $s$ and recover $f$, which is the signal without noise.

There are three threshold rescaling methods available in MATLAB Wavelet Toolbox which are "one", "sln", and "mln". ${ }^{8}$ The scheme "one" follows the basic noise model. The option "sln" corresponds to the basic noise model 

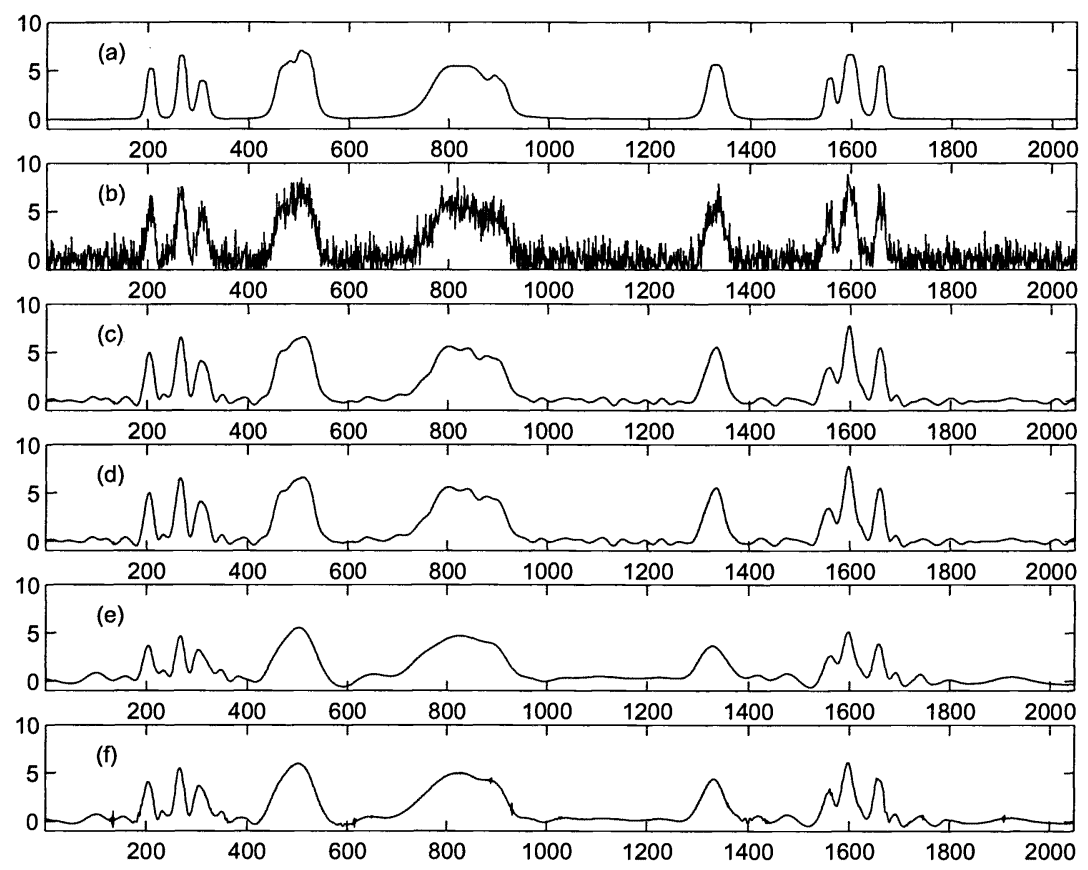

Figure 5. (a) Original signal (b) Noisy signal - Signal to noise ratio $=2$ (c) Denoised signal -heuristic SURE (d) Denoised signal - SURE (e)Denoised signal - Fixed form threshold (f)Denoised signal - Minimax (All are denoised using a Daubechies 10 wavelet with 10 levels of decomposition)

with unscaled noise and performs threshold rescaling using only a single estimation of level noise which is computed based on the first level coefficients of the decomposition. The "mln" method corresponds to the basic noise model with non-white noise and performs threshold rescaling which is based on a level-dependent estimation of the noise at that decomposition level.

\subsection{The Use of Averaging to Denoise}

Averaging is another technique that has been used to reduce noise in heartbeat signals. Heartbeat sounds are quasi-stationary over a short period of time if conditions remain constant. ${ }^{4}$ Averaging in combination with wavelet denoising is examined in the context of how much improvement there is in the SNR and how many cycles should be averaged.

The algorithm for averaging the PCG signal uses the ECG as a gating signal because they are both recorded simulateously. The QRS complex of the ECG signals the beginning of the cycle and is used to separate each heartbeat. A description of the complete algorithm is given by Tinati. ${ }^{4}$

\subsection{Measuring the Results of Denoising}

Signal-to-noise-ratio (SNR) is a traditional method of measuring the amount of noise present in a signal. SNR is defined as $10 * \log _{10}\left(\right.$ Power $_{\text {signal }} /$ Power $\left._{\text {noise }}\right)$ measured in decibels. Two tests are performed using the SNR to measure the performance of wavelet denoising. ${ }^{5}$ Because there is currently no known method to calculate which wavelet and thresholding parameters best denoise a signal, tests must be performed to evaluate the denoising capabilities of wavelets and thresholding parameter combinations. A known amount of noise was added to a "clean" heart sound recording. ("Clean" refers to the fact that although attempts were made to eliminate all environmental noise during the recording, there is still some noise present in small amounts.) Using various parameters, the wavelet denoising technique was applied to the heart sound recording which has noise added. Then the SNR will be calculated for the denoised signal and the original signal. The higher the SNR, the less noise there is present. Another test is to apply 
the wavelet denoising technique to a clean recording and compute the SNR of the resultant signal and the original signal. This test determines how much information in the original signal is lost by the denoising process. In other words, the more of the original clean signal that remains after applying the wavelet transform, the better.

The concept of adding a known amount of noise to clean heartbeats, then denoising the signal, and seeing how much noise remains is also employed to measure how well averaging performs as a denoising technique.

\subsection{Use of the Hilbert Transform}

The Hilbert Transform (HT) may be used to calculate the instantaneous attributes of a signal. The mathematical definition of the Hilbert Transform of is ${ }^{12}$

$$
y(t)=\pi^{-1} \int_{\infty}^{-\infty} \frac{x(\tau)}{t-\tau} d \tau
$$

The Hilbert Transform (HT) can be considered a convolution between the signal and $\frac{1}{\pi} t$. The HT can be realized by an ideal filter whose amplitude response is unity and whose phase response that is a constant ninety degree lag. The HT is called the quadrature filter because it shifts the phase of the spectral components by $\frac{\pi}{2} t$.

Figure 6 borrows the concept of a complex trace from seismic data analysis. ${ }^{13}$ The signal and its Hilbert Transform are projected on their prospective axes with the complex trace being a vector sum of the two. This view reveals many features of the signal. The length of the complex trace vector is the instantaneous amplitude. The orientation angle (usually measured relative to the positive axis of the plane where the real signal is projected) is the instantaneous phase. The time rate of change of the phase angle is the instantaneous frequency which may be seen in Figure 7. The instantaneous frequency is calculated through the analytic method using the Hilbert Transform. The instantaneous frequency is mathematically defined in Equation 5 where $s$ is the signal and $H(s)$ is the Hilbert Transform of the signal. ${ }^{14}$

$$
\Theta=\frac{1}{2 \pi} \frac{d}{d t}\left[\arctan \left(\frac{H[s(t)]}{s(t)}\right)\right]
$$

The instantaneous frequency of a signal may be used to demonstrate how effective denoising is. ${ }^{15}$

It may be seen that the signal is much cleaner after being denoised in Figure 6, but this dramatic improvement is only because the signal has much additive noise. The Hilbert Transform of a signal does not accentuate the noise in a signal and thus does not show such dramatic improval after denoising as is seen in phase space diagrams shown in Maple et al..$^{5}$ The phase space diagrams take the derivative of a signal thus accentuating the noisy, high frequency, content. This fact is demonstrated in Figure 8.

We have demonstrated that the HT has many interesting applications to PCGs. The complex PCG trace reveals much information about the signal. The HT may also be used to calculate the instantaneous parameters of a signal. The phase space diagram better visually demonstrates denoising is instead of plotting the HT and the signal, although instantaneous frequency calculated using the HT effectively demonstrates denoising.

\subsection{Experimental Results}

While no one wavelet seemed to consistently give better results than another, there are a couple recommendations that may be made. The Coiflet 4 and 5, the Daubechies 11, 14, and 20 and the Symlet 9, 11, and 14 seemed to produce slightly better results in terms of noise removal than most of the wavelets tried while the Daubechies 1 , Coiflet 1, and Symlet 1 and 2 seemed to produce marginally worse results.

It appeared that using 5 levels of decomposition usually proved adequate. For three trials (a trial being a clean heartbeat), varying amounts of noise was added $(1 \mathrm{~dB}, 5 \mathrm{dBs}$, and $10 \mathrm{dBs})$ and the levels of decomposition were varied from 1 to 10 with other denoising parameters remaining constant. In most cases, using a decomposition level of 5 , achieved an SNR after denoising within $95 \%$ of the best denoising result for that set of data. However, in a test performed where noise was added to three trials in increments of $1 \mathrm{~dB}$ from 1 to $20 \mathrm{dBs}$, the best denoising results obtained in each case ranged from using 4 to 10 levels of decomposition.

The wavelet transform process of decomposition and recomposition was applied to several clean heartbeats. The SNR of the original signal and the signal after the WT is applied are calculated. This number represents how much information was lost in the wavelet analysis process. Any wavelet that consistently gave an SNR of under $60 \mathrm{dBs}$ 

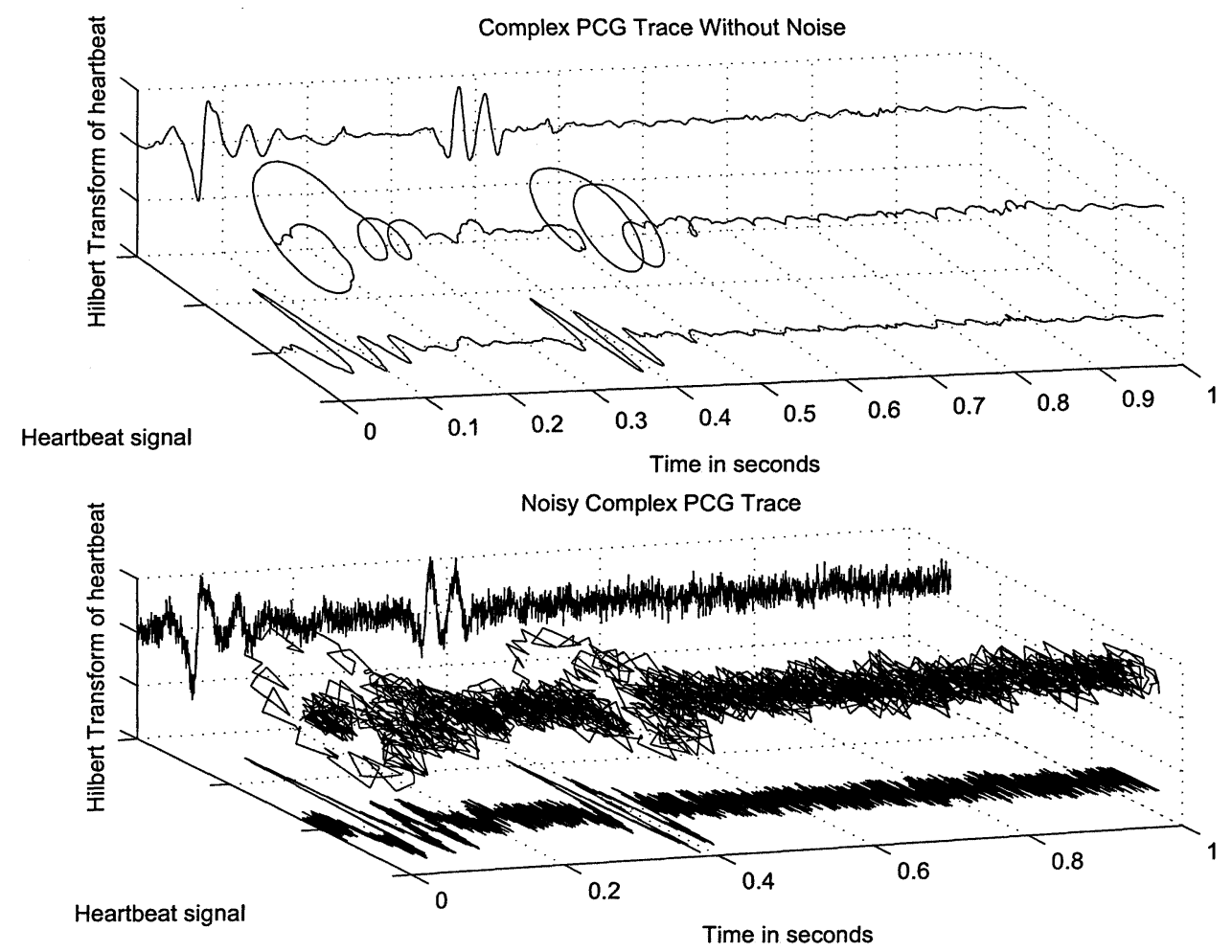

Figure 6. This figure shows a complex PCG trace first with additive white noise and secondly without noise.
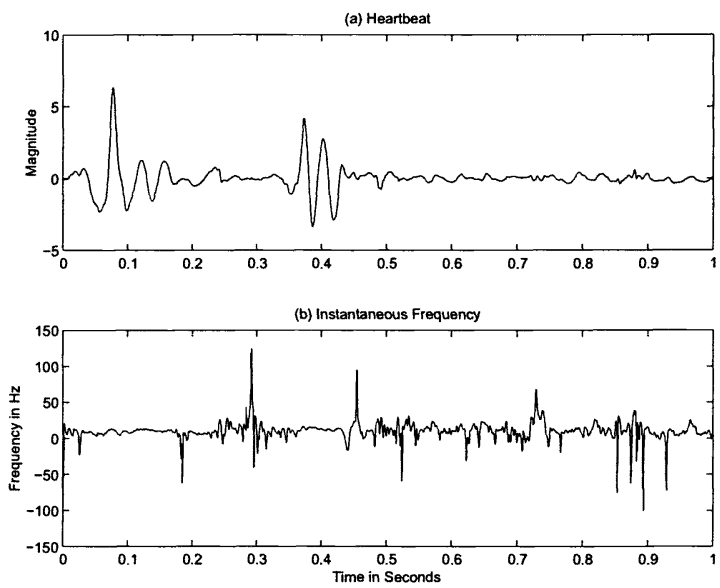

Figure 7. This figure shows (a) a characteristic heartbeat as a reference (b) the instantaneous frequency of a heartbeat. It is interesting to note that at the first and second heart sound the dominant frequency appears to be about $10 \mathrm{~Hz}$. 

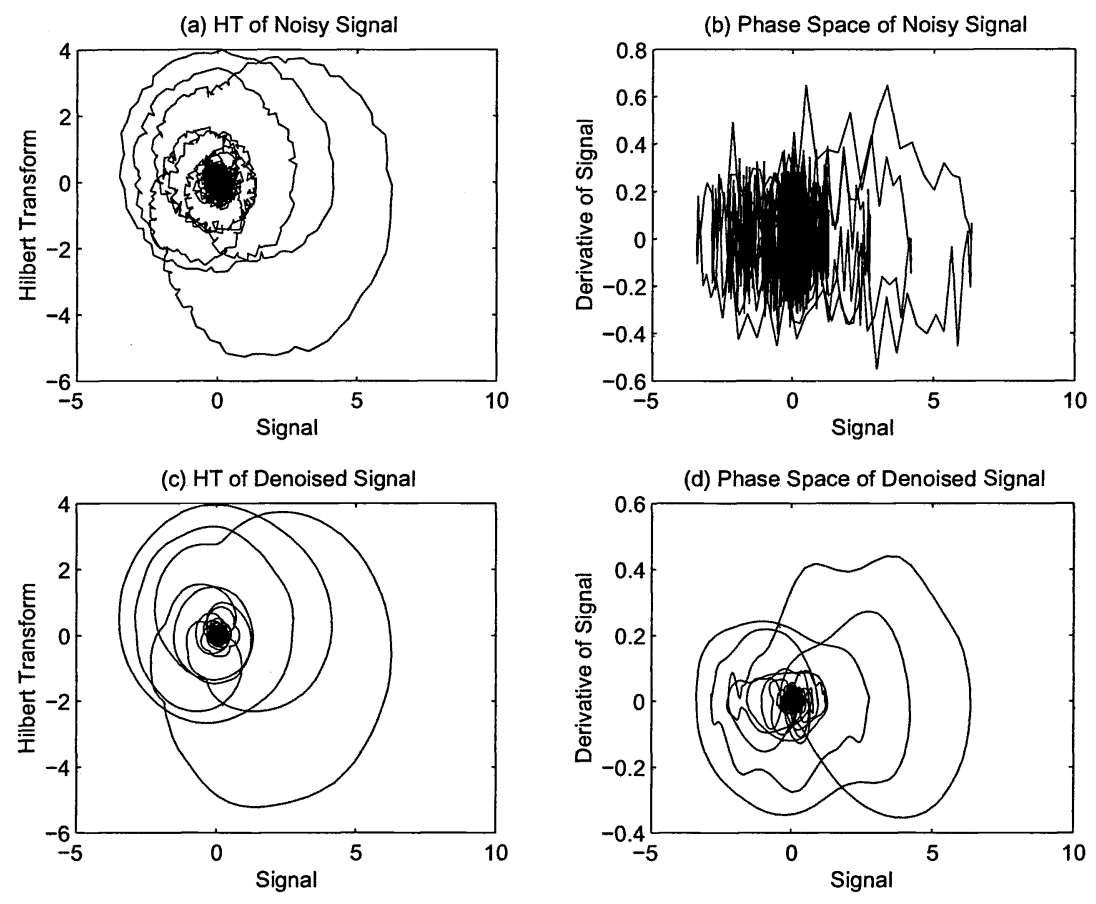

Figure 8. This figure shows (a) HT of a characteristic heartbeat with $20 \mathrm{dBs}$ of additive noise (b) the phase space diagram of the same noisy signal (c) the HT of the denoised heartbeat and (d) the phase space diagram of the denoised heartbeat

was considered to have lost too much information from the original signal. The wavelets which seemed to lose the most information were the Daubechies 1, 2, 3, 43, 44, 45 and the Symlet 1 and 2.

Soft thresholding definitely outperformed hard thresholding in the threshold selection category. Soft thresholding almost always gave better SNR after denoising than hard thresholding. Figure 9 shows how hard thresholding may cause discontinuities in a signal.

Of the four threshold selection rules, the minimax and SURE ("rigrsure") threshold selection schemes are more conservative than the others, and therefore should be used when small details of signal lie in the noise range. The two other schemes remove the noise more aggressively. Because small details of the PCG signal are located in the noise range, it was believed that the "rigrsure" method would be more effective. Our expectations proved to be true. The rigorous SURE method almost always produced better results than the other methods with the heuristic SURE coming in second. Table 4 shows typical results for each of the four threshold selection rules.

The "sln" method performed the best of three threshold rescaling methods available. For example, in Figure 10, with large amounts of noise present, the methods all perform roughly equally with the "sln" method producing slightly better results than the others. However, as less noise is present, the "sln" method appears to be, by far, the best. The "mln" method is a close second. The "one" method gives about the same SNR after denoising no matter what amount of noise was present in the signal.

Figure 11 shows the best results for adding known amounts of noise to three different heartbeats and then applying wavelet denoising with varying parameters. The best results are very similar proving the results of wavelet denoising are easily reproducible for heartbeat sounds. Also, the SNRs after wavelet denoising appear to be relatively linear corresponding to the linear SNR between the signal and the addition of white noise.

Averaging seemed to produce significant improvements especially if there is a large amount of noise present in the signal. Figure 12 shows that averaging a series of 50-75 beats seems to give the best result in terms of recording 

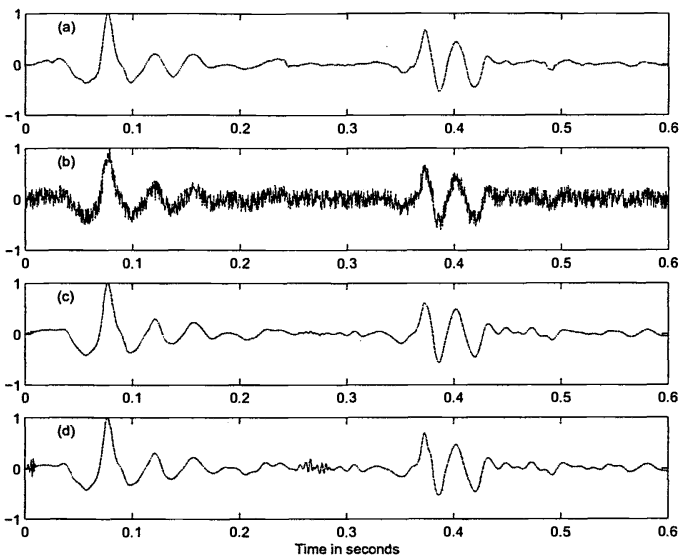

Figure 9. This figure demonstrates that hard thresholding can cause discontinuities in a signal. (a) is characteristic heartbeat signal (b) the heartbeat with white noise added resulting in an SNR of 1 $\mathrm{dB}(\mathrm{c})$ the heartbeat denoised using soft thresholding (d) the heartbeat denoised with hard thresholding

\begin{tabular}{|l|l|l|l|}
\hline $\begin{array}{l}\text { Threshold } \\
\text { Selection } \\
\text { Rule }\end{array}$ & Trial 1 & Trial 2 & Trial 3 \\
\hline "Rigrsure" & 13.65 & 13.78 & 13.70 \\
\hline "Heursure" & 13.27 & 13.24 & 12.72 \\
\hline "Minimaxi" & 9.30 & 8.09 & 9.84 \\
\hline "Sqtwolog" & 7.26 & 8.09 & 7.37 \\
\hline
\end{tabular}

Table 4. Typical SNR results after denoising using four threshold selection rules. White noise was added to three different characteristic heartbeats resulting in an SNR of $1 \mathrm{~dB}$. Wavelet denoising was applied to the heartbeats using the same parameters (Symlet 14 wavelet with 10 levels of decomposition, soft thresholding, and used the basic noise model with a single estimation level of noise) except for varied threshold selection rules. The SNR was then calculated in dBs. The "rigrsure" outperforms all other methods in every case.

and computation time tradeoff. It is difficult to obtain a long, clean recording and also increases the computation time required.

Figure 13 shows a comparison of using wavelet denoising only and wavelet denoising combined with averaging. It clearly demonstrates that combining the techniques is much more effective. It shows that given a choice between averaging 30,60 or 90 beats that 60 beats provides a good compromise in terms of denoising and recording and computation time.

\section{CONCLUSIONS}

We have demonstrated that wavelet denoising techniques and averaging are useful for removing white noise from heart sounds. Wavelet denoising in combination with averaging produced the best denoising results of the methods applied. However, there may be certain clinical cases, for example if a pathological condition was only present in some beats and not others, where wavelet denoising alone should be employed as averaging reduces a sequence of heartbeats to a single characteristic heartbeat.

Although there was no evidence that a single wavelet was the best suited for denoising heartbeats, there were some wavelets which were slightly better than others and certain wavelets which would not be recommended for this purpose. We reached the conclusion that a decomposition level of 5 produced reasonable results while decomposing the signal further often produced marginal benefits and increases computation time. Soft threshold definitively outperformed hard thresholding. Of the four threshold selection rules, the "rigrsure" rule performed the best, and the best choice of the threshold rescaling methods proved to be the "sln" method. Averaging heartbeats to produce a characteristic heartbeat significantly reduced noise. Averaging a sequence of about 60 heartbeats gave optimal denoising benefits in terms of computation time and difficulty in recording a clean PCG.

The use of the Hilbert Transform was explored in relation to the analysis of heartbeats. Much information is displayed in the complex PCG trace. The instantaneous frequency of a PCG, as a tool of clinical use which perhaps would reveal information about the condition of the heart, merits further research.

Future topics to research in this area of phonocardiogram denoising include the use of wavelet packets, which uses the same principle as wavelet analysis but decomposes both the details and the approximations of the signal at each level resulting in a tree like structure and then chooses the best tree structure for thresholding based on entropy, ${ }^{8}$ and the use of the matching pursuit method. ${ }^{16}$ 


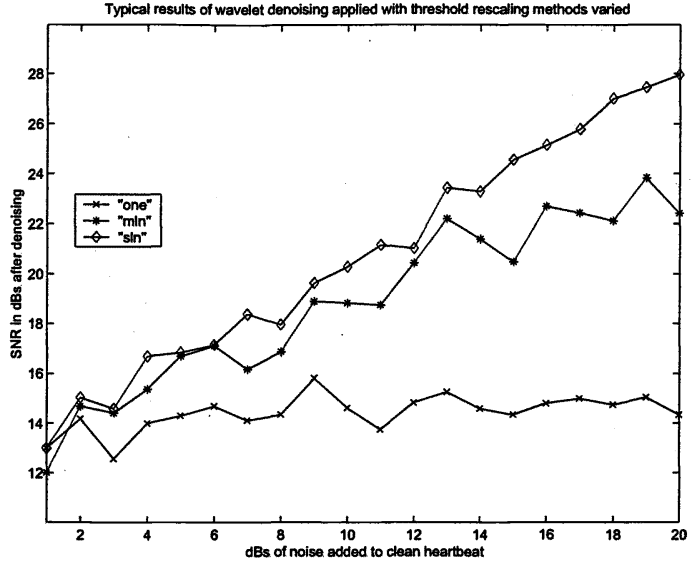

Figure 10. This figure is a comparison of the different threshold rescaling methods. Noise in increments of 1 $\mathrm{dB}$ from 1 to $20 \mathrm{dBs}$ is added to a clean characteristic heartbeat. The signal is then denoised using wavelet denoising (Daubechies 14 wavelet with 10 levels of decomposition using soft thresholding, and the rigorous SURE threshold selection rule) varying the threshold rescaling methods. Overall, the "sln" method performs the best.

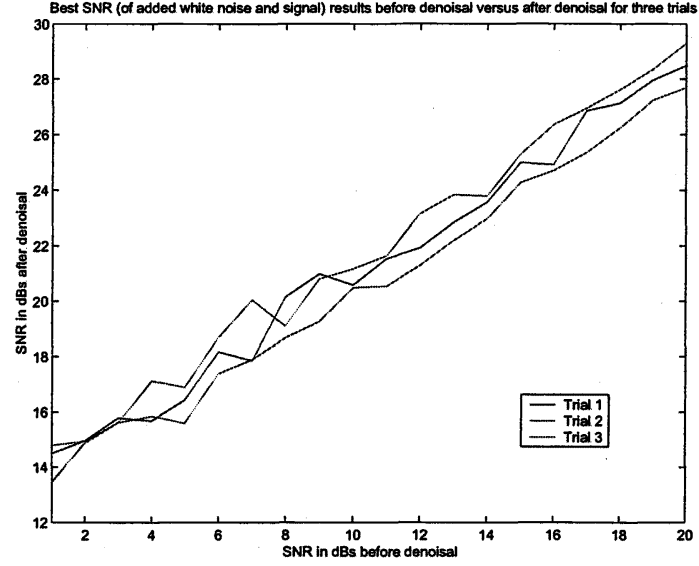

Figure 11. Best SNR results before denoising versus after wavelet denoising for three trials. Each trial is a different, clean heartbeat. A known amount of white noise in decibels is added to a characteristic heartbeat. Different combinations of wavelets, thresholding techniques, and levels of decomposition are tried. The highest SNR after denoising if displayed for each trial. It is interesting to note that the best results for each trial are very similar.

\section{ACKNOWLEDGMENTS}

We would like to thank Leonard Hall, Jarrad Maple and Mohammed Ali Tinati (all of the Department of Electrical and Electronic Engineering, The University of Adelaide) for allowing us to make use of their heart sound recording equipment and software.

We would also like to thank colleagues at the Centre for Biomedical Engineering (Electrical and Electronic Engineering, The University of Adelaide) including Brad Ferguson, Leonard Hall, Greg Harmer, and Samuel Mickan, for providing help and inputs in various areas along the way.

\section{REFERENCES}

1. L.-G. Durand and P. Pibarot, "Digital signal processing of the phonocardiogram: Review of the most recent advancements," Critical Reviews in Biomedical Engineering 23(3/4), pp. 163-219, 1995.

2. S. Lukkarinen, A. Nopanen, K. Sikio, and A. Angerla, "A new phonocardiographic recording system," Computers in Cardiology 27, pp. 117-120, 1997.

3. S. Lukkarinen, K. Sikio, A.-L. Nopanen, A. Angerla, and R. Sepponen, "Novel software for real-time processing of phonocardiographic signal," in Proceedings of the 19th International Conference of the IEEE Engineering in Medicine and Biology Society, pp. 1455-1457, IEEE Engineering in Medicine and Biology Society, October/November 1997.

4. M. A. Tinati, Time-Frequency and Time-Scale Analysis of Phonocardiograms with Coronary Artery Disease Before and After Angioplasty. PhD thesis, The University of Adelaide, October 1998.

5. J. Maple, L. Hall, J. Agzarian, and D. Abbott, "Sensor system for heart sound biomonitor," Proc. SPIE Electronics and Structures for MEMS 3891, pp. 99-109, October 1999.

6. R. Polikar, "The wavelet tutorial." http://www.public.iastate.edu/ rpolikar/WAVELETS/waveletindex.html, August 2000.

7. B. B. Hubbard, The World According to Wavelets, A K Peters Ltd., 289 Linden Street, Wellesley, MA 02181, 1996. 


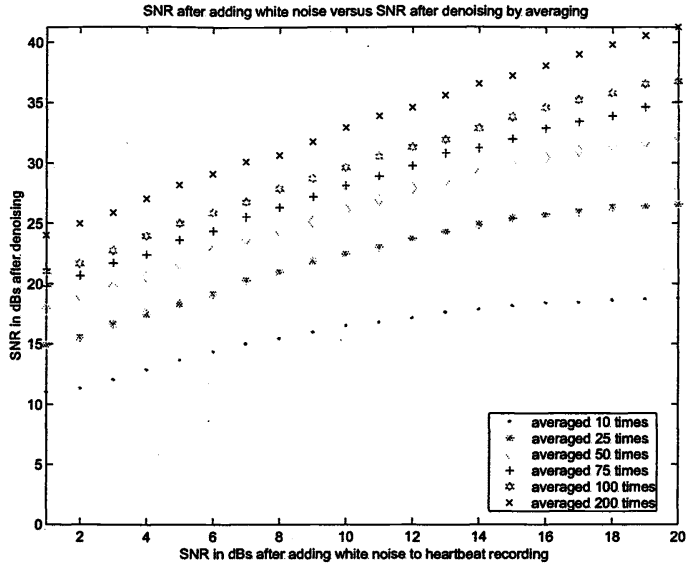

Figure 12. This chart shows the SNR after adding white noise to a series of heartbeats and the SNR after averaging these series of heartbeats to obtain a characteristic heartbeat and reduce noise. There seems to be marginal improvement in SNR when little noise is present in the signal and the signal is not averaged a fair number of times. For example, with an SNR of 1 $\mathrm{dB}$, after averaging the signal 10 times, we see the noise levels decrease as the SNR approaches $11 \mathrm{dBs}$, but with an initial SNR of $20 \mathrm{dBs}$ and averaging 10 heartbeats, the SNR is still about $20 \mathrm{dBs}$ meaning the noise remains. Averaging a series of $50-75$ beats seems to give the best results here in terms of recording and computation time tradeoff.

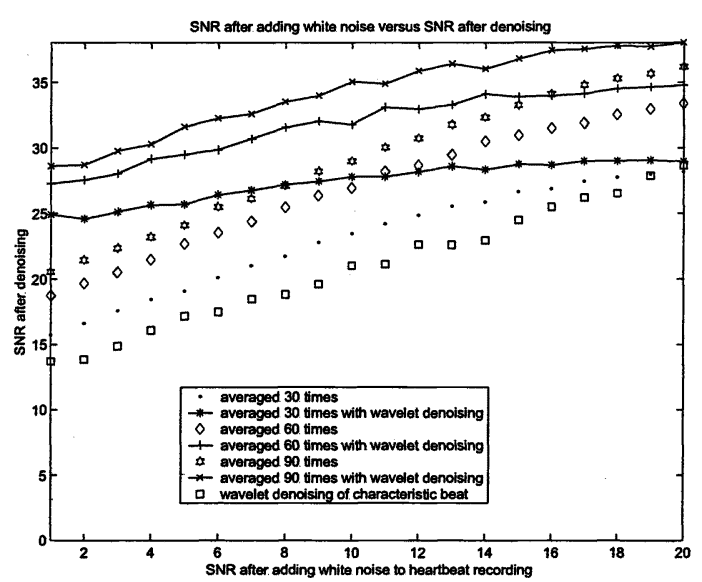

Figure 13. This graph shows the SNR after adding white noise to a series of heartbeats versus the SNR after denoising the signal. Various methods are tried: wavelet denoising only, averaging only, and wavelet denoising combined with averaging. The wavelet denoising combined with averaging is the most successful denoising method. Heartbeat signals of lengths of 30,60 and 90 were averaged. It appears that averaging at least 60 heartbeats would be recommended. There is a significant improval of averaging 60 beats over 30 heartbeats while averaging 90 beats over 60 beats does not show nearly the same improvement.

8. M. Misiti, Y. Misiti, G. Oppenheim, and J.-M. Poggi, Wavelet Toolbox: For Use With Matlab. The Math Works Inc., 1996.

9. S. Mallat, "A theory for multiresolution signal decomposition: the wavelet representation," IEEE Pattern Anal. and Machine Intell. 11(7), pp. 674-693, 1989.

10. C. S. Burrus, R. A. Gopinath, and H. Guo, Introduction to Wavelets and Wavelet Transforms: A Primer, Prentice-Hall, Inc., Upper Saddle River, New Jersey 07458, 1998.

11. M. Akay, "Wavelet applications in medicine," IEEE Spectrum 34, pp. 50-56, May 1997.

12. O. Ersoy, Fourier-Related Transforms, Fast Algorithms and Applications, Prentice-Hall, Inc., Upper Saddle River, New Jersey 07458, 1997.

13. M. T. Taner, F. Koehler, and R. E. Sheriff, "Complex seismic trace analysis," Geophysics 44, pp. 1041-1063, June 1979.

14. J. Gao, X. Dong, W.-B. Want, Y. Li, and C. Pan, "Instantaneous parameters extraction via wavelet transform," IEEE Transactions on Geoscience and Remote Sensing 37, pp. 867-870, March 1999.

15. P. Carré, H. Leman, C. Fernandez, and C. Marque, "Denoising of the uterine ehg by an undecimated wavelet transform," IEEE Transactions on Biomedical Engineering 45, pp. 1104-1113, September 1998.

16. S. Mallat and Z. Zhang, "Matching pursuits with time-frequency dictionaries," IEEE Transactions on Signal Processing 41, pp. 3397-3415, December 1993. 